\title{
Antiplane Problems of Monoclinic Material
}

\author{
By Chien-Ching $\mathrm{Ma}^{1}$
}

\begin{abstract}
Aвstract: The antiplane problem of anisotropic materials is studied by the Mellin transform technique. The material considered in this study possesses a symmetry plane at $z=0$. The general solutions of the anisotropic case are obtained in the Mellin transform domain, which are found to have a similar form as for the isotropic case. The exact full-field solutions for stresses and displacement of the boundaryvalue problem for anisotropic bimaterial wedge subjected to prescribed traction on the wedge surfaces are investigated in detail. The asymptotic behavior of the stress in the vicinity of the apex for an anisotropic bimaterial wedge is presented. The field solutions of interfacial crack along two anisotropic materials and a single anisotropic wedge subjected to a pair of concentrated point loads are expressed explicitly by infinite series. This solution is the Green's function for more general problems. The results show that the stress and displacement fields have reduced dependence on the elastic constants, significantly reducing the algebraic complexity of the solution.
\end{abstract}

\section{INTRODUCTION}

Over the past years, many authors have considered the problem of determining the stress distribution around defects. The strength of materials is influenced by existing defects such as cracks, which can cause a stress concentration near the defects. The existence of an inverse-square-root singularity around the crack tip in an elastic homogeneous medium is well known. Problems related to stress singularities have received much attention in the theory of elasticity. The problem of finding stress singularities at the apex of an isotropic elastic wedge was considered by Williams (1952) by using the eigenfunction-expansion method. Tranter (1948) used the Mellin transform in conjunction with the Airy stress function of plane elasticity to solve the isotropic wedge problem. The problem of a composite wedge consisting of two isotropic elastic materials has received considerable attention since the 1960 s. Lightweight composite materials have been used in the aerospace industries for many years. The most important design information for these materials is the failure criteria, which require rigorous stress analysis at the singular point. Williams (1959) used an eigenfunction approach to establish the asymptotic nature of the dominant singular stresses of dissimilar materials with a semiinfinite crack. He found that the stresses share the inverse-square-root singularity of the crack in a homogeneous material and, in addition, exhibit an oscillatory behavior as the crack tip is approached. Following Williams's study on bimaterial interfacial crack problems, Erdogan (1963), England (1965), and Rice and Sih (1965) tried to obtain the solutions with the Muskhelishili's complex function theory in elasticity. Bogy (1971) and Ma and Wu (1990) used the Mellin transform to treat the problem of two dissimilar isotropic elastic wedges of arbitrary angles. The stress field at the vertex of the edge or the corner of the elastic bimaterial wedge possesses a singularity, the nature of which depends on the composite parameters of the material combination. Dundurs (1967)

1Prof., Dept. of Mech. Engrg., Nat. Taiwan Univ., Taipei, Taiwan 10764, R.O.C.

Note. Discussion open until February 1, 1993. To extend the closing date one month, a written request must be filed with the ASCE Manager of Journals. The manuscript for this paper was submitted for review and possible publication on September 20, 1991. This paper is part of the Journal of Engineering Mechanics, Vol. 118, No. 9, September, 1992. (C)ASCE, ISSN 0733-9399/92/0009-1765/\$1.00 + $\$ .15$ per page. Paper No. 2574 . 
derived two composite parameters from the elastic constants of the materials and showed that the stress field of a composite in a state of plane deformation depends only on these two parameters. Solutions of this classical problem are unsatisfactory in the sense that the oscillations are in the singular stress field. Several modifications have been suggested to resolve this problem, such as the contact-zone model of Comninou (1977) and the cohesive zone model of Atkinson (1977).

Investigation of the analogous wedge problems for anisotropic materials are motivated by the increasingly important application of these materials in modern technology. In real composite materials, each layer is fiberreinforced laminated material, and hence the composite should be regarded as an anisotropic material. Stroh (1958) obtained an analytical expression for the stresses near the tip of a plane crack in an anisotropic material of infinite extent. Following the approach of Stroh, Ting (1986) studied the stress distribution in a composite wedge of anisotropic materials. Bogy (1972) employed a complex-function representation of a generalized Mellin transform to analyze stress singularities in an anisotropic wedge. All these studies have shown that anisotropic media retain the troublesome feature of oscillation for the singular stress field observed in isotropic media. Ma and Hour (1989) considered the antiplane problem of two dissimilar anisotropic wedges of arbitrary angles that are bonded together perfectly along a common edge. $\mathrm{Ma}$ and Hour (1989) found that the order of the stress singularity is always real for antiplane dissimilar anisotropic wedge problems, which is of a quite different character from the in-plane case, in which the complex type of stress singularity might exist. Other investigations of interface cracks of anisotropic solids are Gotoh (1967), Willis (1971), Qu and Bassani (1989), and Wu $(1990,1991)$.

Solutions of antiplane problems serve two distinct purposes. First, they may be used to shed some light on the qualitative behavior of the solutions of more difficult in-plane problems. Second, they may have practical applications in their own right in situations such as three-dimensional problems in which the third mode is separable. Most of the studies of dissimilarmaterial problems just mentioned focused only on the determination of the stress singularity. In the present investigation, we are able to construct the exact full-field solutions for the anisotropic bimaterial wedge subjected to prescribed tractions. The material considered in this study is a monoclinic material that has a symmetry plane at $z=0$ but is otherwise arbitrary. In this paper, the Mellin transform method, originally applied to the analysis of wedge problems by Tranter (1948) and extended to study the anisotropic material by Bogy (1972), is used to analyze the antiplane problem of anisotropic material. It is shown that solutions for the stress and displacement fields have a reduced dependence on the elastic constants. The reduction in the number of elastic constants simplifies the description of the stress and displacement state. In the section headed "General Solutions," the general solution of the governing equation in Mellin transform domain is obtained and is found to have similar functional form as that for the isotropic material. In the section headed "Full-Field Solutions," we consider two dissimilar elastic anisotropic homogeneous materials that are perfectly bonded along an interface that is either plane or has a radius of curvature sufficiently large so that the configuration can be approximated by that of two bonded wedges of arbitrary angles. The mathematical model that is analyzed for this problem is shown in Fig. 1. The composite is assumed to be loaded along the boundary of the wedge surface $B$ and $B^{*}$. The region above the interface is occupied 


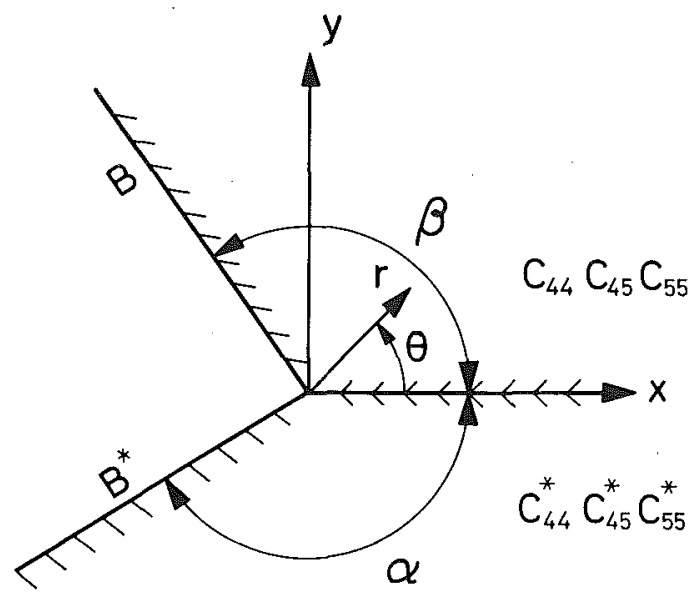

FIG. 1. Configuration of Bonded Antiplane Anisotropic Dissimilar Wedges

by the first material, with material constants $c_{44}, c_{45}$, and $c_{55}$, and the region below the interface is occupied by the second material, with material constants $c_{44}^{*}, c_{45}^{*}$, and $c_{55}^{*}$. The problem is solved by making use of the theory of residues to obtain the explicit full-field solutions of stresses and displacement. In the section headed "Green's Functions," the analytical explicit solutions for stresses and displacement are expressed in power series form for an interfacial crack and a single homogeneous wedge subjected to a pair of concentrated point loads. It is found that the full stress field of the interfacial crack is independent of material constants if the loadings applied on upper and lower crack surface have the same function forms.

\section{General Solutions in Mellin Transform Domain}

\section{Isotropic Case}

We consider the isotropic material first. By definition the only nonvanishing displacement component $w$ is along $z$-axis for the antiplane deformation. The equilibrium equation for the nonvanishing displacement $w$ is given by the following partial differential equation:

$\frac{\partial^{2} w}{\partial r^{2}}+\frac{1}{r} \frac{\partial w}{\partial r}+\frac{1}{r^{2}} \frac{\partial^{2} w}{\partial \theta^{2}}=0$

The nonvanishing stresses are

$\tau_{r z}=\mu \frac{\partial w}{\partial r}$

$\tau_{\theta z}=\frac{\mu}{r} \frac{\partial w}{\partial \theta}$

Let the Mellin transform of a function $f(r)$, defined and suitably regular on $0<r<\infty$, be denoted by $\hat{f}(s)$

$\hat{f}(s)=M\{f ; s\}=\int_{0}^{\infty} f(r) r^{s-1} d r$ 
where $s=$ a complex transform parameter. Let $\hat{w}(s, \theta), \hat{\tau}_{r z}(s, \theta)$, and $\hat{\tau}_{\theta z}(s, \theta)$ in this order denote the Mellin transforms of $w(r, \theta), r \tau_{r z}(r, \theta)$, and $r \tau_{\theta z}(r, \theta)$ with respect to $r$. Accordingly

$\hat{w}(s, \theta)=\int_{0}^{\infty} w(r, \theta) r^{s-1} d r$

$\hat{\tau}_{r z}(s, \theta)=\int_{0}^{\infty} \tau_{r z}(r, \theta) r^{s} d r$

$\hat{\tau}_{\theta z}(s, \theta)=\int_{0}^{\infty} \tau_{\theta z}(r, \theta) r^{s} d r$

Application of the inversion formula to (5)-(7) gives

$w(r, \theta)=\frac{1}{2 \pi i} \int_{\rho-i \infty}^{\rho+i \infty} \hat{w}(s, \theta) r^{-s} d s$

$\tau_{r z}(r, \theta)=\frac{1}{2 \pi i} \int_{p-i \infty}^{\rho+i \infty} \hat{\tau}_{r z}(s, \theta) r^{-s-1} d s$

$\tau_{\theta z}(r, \theta)=\frac{1}{2 \pi i} \int_{\rho-i \infty}^{\rho+i \infty} \hat{\tau}_{\theta z}(s, \theta) r^{-s-1} d s$

The path of integration in the complex line integrals $\operatorname{Re}(s)=\rho$ in (8), (9), and (10) must lie within a common strip of regularity of their integrands; the choice of $\rho$ is taken to be

$\rho=-\varepsilon \quad 0<\varepsilon<\left(\left|\operatorname{Re}\left(s_{1}\right)\right|\right)$

where $s_{1}$ denotes the location of the pole in the open strip $-1<\operatorname{Re}(s)<$ 0 with the largest real part; and Re denotes the real part of the complex argument, as shown in Fig. 2.

Application of the Mellin transform (5) to (1) yields an ordinary differential equation for $\hat{w}$, the general solution of which is

$\hat{w}(s, \theta)=a(s) \sin (s \theta)+b(s) \cos (s \theta)$

in which the functions $a(s)$ and $b(s)$ are to be determined through the boundary conditions. The general expressions for the transformed shear stresses are

$\hat{\tau}_{\theta z}(s, \theta)=\mu s[a(s) \cos (s \theta)-b(s) \sin (s \theta)]$
$\hat{\tau}_{r z}(s, \theta)=-\mu s[a(s) \sin (s \theta)+b(s) \cos (s \theta)]$

\section{Anisotropic Case}

Next, the general solution in the Mellin transform domain for anisotropic material is constructed. The method employs the complex representation of the antiplane anisotropic elasticity solution in conjunction with a generalization of the Mellin transform. If the plane of elastic symmetry is assumed to be normal to the $z$-axis, then there are only three relevant coefficients $\left(c_{44}, c_{45}\right.$, and $\left.c_{55}\right)$ to be considered. The stress components are related to the displacement as follows:

$\tau_{y z}=c_{44} \frac{\partial w}{\partial y}+c_{45} \frac{\partial w}{\partial x}$ 


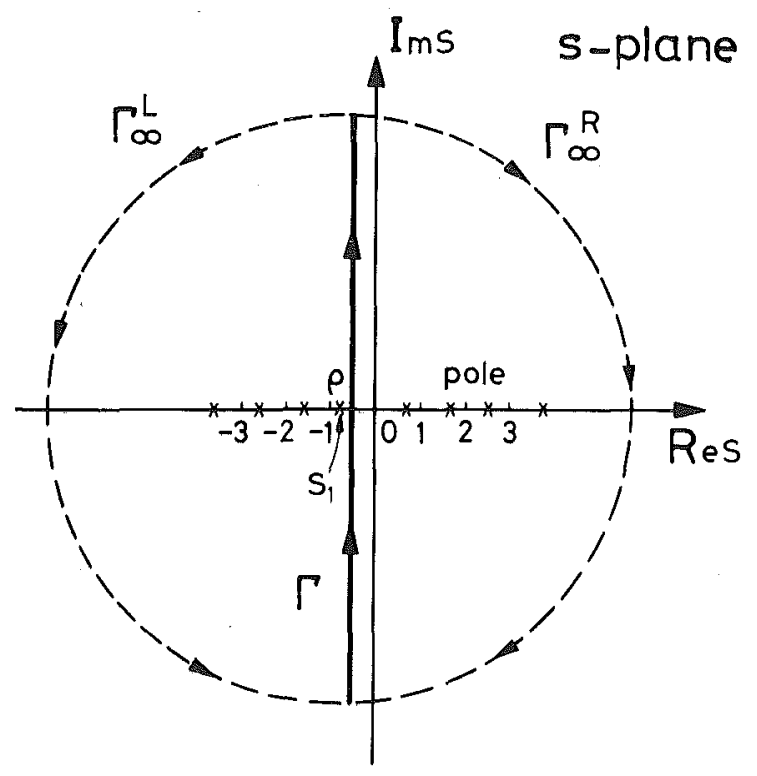

FIG. 2. Path of Integration Used in Complex s-Plane

$\tau_{x z}=c_{45} \frac{\partial w}{\partial y}+c_{55} \frac{\partial w}{\partial x}$

In the absence of body forces, the corresponding displacement equation of equilibrium for a homogeneous anisotropic material is given by

$c_{55} \frac{\partial^{2} w}{\partial x^{2}}+2 c_{45} \frac{\partial^{2} w}{\partial x \partial y}+c_{44} \frac{\partial^{2} w}{\partial y^{2}}=0$

The governing equation (17) can be solved in the complex plane $\xi=x+$ py such that

$w(x, y)=2 \operatorname{Re}[U(\xi)]$

where $U=$ an arbitrary function of $\xi$; and $p=$ a value that depends on the material constants. Substitution of (18) into (17) yields the following characteristic equation for $p$ :

$c_{44} p^{2}+2 c_{45} p+c_{55}=0$

Hence

$p=\frac{-c_{45} \pm i \sqrt{c_{44} c_{55}-\left(c_{45}\right)^{2}}}{c_{44}}$

Following Ma and Hour (1989), the general solution of the equilibrium equation for an anisotropic material in the Mellin transform domain can be simplified as follows:

$\hat{w}(s, \theta)=\Psi^{-s}(\theta)[a(s) \sin s \phi+b(s) \cos s \phi]$

$\hat{\tau}_{\theta z}(s, \theta)=C s \Psi^{-s}(\theta)[a(s) \cos s \phi-b(s) \sin s \phi]$ 
$\hat{\tau}_{r z}(s, \theta)=C s \Psi^{-(s+2)}(\theta)[a(s)(\Theta \cos s \phi-\Omega \sin s \phi)$

$-b(s)(\Theta \sin s \phi+\Omega \cos s \phi)]$

where

$C=\left[c_{44} c_{55}-\left(c_{45}\right)^{2}\right]^{1 / 2}$

$\tan \phi=\frac{\sqrt{c_{44} c_{55}-\left(c_{45}\right)^{2}} \sin \theta}{c_{44} \cos \theta-\mathbf{c}_{45} \sin \theta}$

$\Psi(\theta)=\left[\cos ^{2} \theta-\frac{c_{45}}{c_{44}} \sin 2 \theta+\frac{c_{55}}{c_{44}} \sin ^{2} \theta\right]^{1 / 2}$

$\Theta=\sin \theta \cos \theta+\frac{c_{45}}{c_{44}} \cos 2 \theta-\frac{c_{55}}{c_{44}} \sin \theta \cos \theta$

$\Omega=\frac{\sqrt{c_{44} c_{55}-\left(c_{45}\right)^{2}}}{c_{44}}$

If comparison is made of the general solution of $\hat{w}$ and $\hat{\tau}_{\theta z}$ in (20) and (21) for an anisotropic material to those for the isotropic material as presented in (12) and (13), a very simple relationship is obtained. The solution for an anisotropic material can be obtained by replacing the shear modulus $\mu$ for an isotropic material by $C$ and angle $\theta$ by $\phi$ and times $\Psi^{-s}(\theta)$. We shall refer to $C$ as the effective material constant, and $\phi$ as the effective angle. A similar conclusion was also reached by $\mathrm{Wu}(1991)$. With the general solution (20)-(22a) at hand, we are able to solve any antiplane anisotropic problem subjected to different boundary conditions. For the application of the results just obtained, the problem of anisotropic bimaterial wedges with arbitrary angle is solved in next section.

\section{Full-Field Solutions for Anisotropic Bimaterial Wedges}

The difference in the elastic properties of the components in a composite causes high stress concentrations at the edges of the interface. The high stress concentrations may result in partial debonding or initiation of an interfacial failure. Consider two anisotropic wedges with arbitrary angles that are assumed to be perfectly bonded together along a common edge as shown in Fig. 1. The interface lies along the positive $x$-axis with the anisotropic material constants $c_{44}, c_{45}$, and $c_{55}$ lying above and $c_{44}^{*}, c_{45}^{*}$, and $c_{55}^{*}$ material constants below. The bimaterial wedge is subjected to surface tractions acting in edges $B$ and $B^{*}$. The traction boundary conditions are given as follows:

$\tau_{\theta z}(r, \beta)=t(r)$

$\tau_{\theta z}^{*}(r,-\alpha)=t^{*}(r)$

where $t(r)$ and $t^{*}(r)$ represent the prescribed shearing tractions on $B$ and $B^{*}$ and are assumed to be continuously differentiable and absolutely integrable on $0 \leq r<\infty$. Thus, the following integrals exist: $\int_{0}^{\infty}|t(r)| d r ; \int_{0}^{\infty}$ $\left|t^{*}(r)\right| d r$. Because of the assumed regularity of the prescribed load functions $t(r)$ and $t^{*}(r)$ on $0 \leq r<\infty$, they satisfy $t(r), t^{*}(r)=O\left(r^{-1}\right)$ as $r \rightarrow \infty$.

Assuming the interface is perfectly bonded, one can immediately establish 
the continuity conditions of the stress and displacement along the interface as follows:

$$
\begin{aligned}
& \tau_{\theta z}(r, 0)=\tau_{\theta z}^{*}(r, 0) \\
& w(r, 0)=w^{*}(r, 0)
\end{aligned}
$$

In addition, we shall require the stress components of the stress fields to satisfy the regularity condition $\tau_{\theta z}, \tau_{r z}=O\left(r^{-1+\eta}\right)$ as $r \rightarrow \infty$ for every $\eta>$ 0 .

The exact solutions of stresses and displacement in the Mellin transform domain that satisfy boundary and continuity conditions (23) and (24) are $(0 \leq \theta \leq \beta)$

$$
\begin{aligned}
& \hat{\tau}_{\theta z}(s, \theta)=\frac{1}{\Psi^{s}(\theta) D(s)}\left[\hat{t}(s) \Psi^{s}(\beta)\left(K \sin s \phi_{\alpha} \cos s \phi+\cos s \phi_{\alpha} \sin s \phi\right)\right. \\
& \left.+\hat{t}^{*}(s) \Psi^{s}(-\alpha)\left(\sin s \phi_{\beta} \cos s \phi-\cos s \phi_{\beta} \sin s \phi\right)\right] \ldots \ldots \ldots \ldots \ldots
\end{aligned}
$$

$\hat{\tau}_{r z}(s, \theta)=\frac{1}{\Psi^{s+2}(\theta) D(s)}\left\{\hat{t}(s) \Psi^{s}(\beta)\left[K \sin s \phi_{\alpha}(\Theta \cos s \phi-\Omega \sin s \phi)\right.\right.$

$\left.+\cos s \phi_{\alpha}(\Theta \sin s \phi+\Omega \cos s \phi)\right]+\hat{t}^{*}(s) \Psi^{s}(-\alpha)\left[\sin s \phi_{\beta}(\Theta \cos s \phi\right.$

$\left.\left.-\Omega \sin s \phi)-\cos s \phi_{\beta}(\Theta \sin s \phi+\Omega \cos s \phi)\right]\right\}$

$\hat{w}(s, \theta)=\frac{1}{C s \Psi^{s}(\theta) D(s)}\left[\hat{t}(s) \Psi^{s}(\beta)\left(K \sin s \phi_{\alpha} \sin s \phi-\cos s \phi_{\alpha} \cos s \phi\right)\right.$

$\left.+\hat{t}^{*}(s) \Psi^{s}(-\alpha)\left(\sin s \phi_{\beta} \sin s \phi+\cos s \phi_{\beta} \cos s \phi\right)\right]$

and for $-\alpha \leq \theta \leq 0$

$$
\hat{\tau}_{\theta z}^{*}(s, \theta)=\frac{K}{\Psi^{* s}(\theta) D(s)}
$$

$\left[\hat{t}(s) \Psi^{s}(\beta)\left(\sin s \phi_{\alpha} \cos s \phi^{*}+\cos s \phi_{\alpha} \sin s \phi^{*}\right)+\hat{t}^{*}(s) \Psi^{s}(-\alpha)\right.$

$\left.\left(\frac{1}{K} \sin s \phi_{\beta} \cos s \phi^{*}-\cos s \phi_{\beta} \sin s \phi^{*}\right)\right]$

$\hat{\tau}_{r z}^{*}(s, \theta)=\frac{K}{\Psi^{*(s+2)}(\theta) D(s)}\left\{\hat{t}(s) \Psi^{s}(\beta)\left[\sin s \phi_{\alpha}\left(\Theta^{*} \cos s \phi^{*}\right.\right.\right.$

$\left.\left.-\Omega^{*} \sin s \phi^{*}\right)+\cos s \phi_{\alpha}\left(\Theta^{*} \sin s \phi^{*}+\Omega^{*} \cos s \phi^{*}\right)\right]$

$+\hat{t}^{*}(s) \Psi^{s}(-\alpha)\left[\sin s \phi_{\beta} \frac{\Theta^{*} \cos s \phi^{*}-\Omega^{*} \sin s \phi^{*}}{K}\right.$

$\left.\left.-\cos s \phi_{\beta}\left(\Theta^{*} \sin s \phi^{*}+\Omega^{*} \cos s \phi^{*}\right)\right]\right\}$ 
$\hat{w}^{*}(s, \theta)=\frac{1}{C s \Psi^{* s}(\theta) D(s)}$

$\cdot\left[\hat{t}(s) \Psi^{s}(\beta)\left(\sin s \phi_{\alpha} \sin s \phi^{*}-\cos s \phi_{\alpha} \cos s \phi^{*}\right)+\hat{t}^{*}(s) \Psi^{s}(-\alpha)\right.$

$\left.\cdot\left(\frac{\sin s \phi_{\beta} \sin s \phi^{*}}{K}+\cos s \phi_{\beta} \cos s \phi^{*}\right)\right]$

in which

$\Psi(\beta)=\left(\cos ^{2} \beta-\frac{c_{45}}{c_{44}} \sin 2 \beta+\frac{c_{55}}{c_{44}} \sin ^{2} \beta\right)^{1 / 2}$

$\Psi(-\alpha)=\left(\cos ^{2} \alpha+\frac{c_{45}^{*}}{c_{44}^{*}} \sin 2 \alpha+\frac{c_{55}^{*}}{c_{44}^{*}} \sin ^{2} \alpha\right)^{1 / 2}$

$\tan \phi_{\beta}=\frac{\sqrt{c_{44} c_{55}-c_{45}^{2}} \sin \beta}{c_{44} \cos \beta-c_{45} \sin \beta}$

$\tan \phi_{\alpha}=\frac{\sqrt{c_{44}^{*} c_{55}^{*}-c_{45}^{* 2}} \sin \alpha}{c_{44}^{*} \cos \alpha+c_{45}^{*} \sin \alpha}$

$K=\frac{C^{*}}{C}$

and

$D(s)=K \sin s \phi_{\alpha} \cos s \phi_{\beta}+\sin s \phi_{\beta} \cos s \phi_{\alpha}$

The function $\phi^{*}, \Theta^{*}, \Omega^{*}$, and $C^{*}$ have the same functional form of $\phi, \Theta$, $\Omega$, and $C$ except $c_{44}, c_{45}$, and $c_{55}$ are replaced by $c_{44}^{*}, c_{45}^{*}$, and $c_{55}^{*} ; \hat{t}(s)$ and $\hat{t}^{*}(s)$ denote the Mellin transforms of $t(r)$ and $t^{*}(r)$, respectively. The function $D(s)$ is the characteristic equation that gives important information concerning the behavior of the stresses and displacement.

We can now use the inversion integrals in (8)-(10) to obtain the full-field solutions of the stresses and displacement. The integration path is chosen to lie within the strip of regularity $\operatorname{Re}\left(s_{1}\right)<\rho<0$, with $s_{1}$ denoting the pole with the largest real part of (25)-(30) in the strip $-1<\operatorname{Re}(s)<0$. Applying Cauchy's integral theorem, the value of the inversion integral along $\Gamma$ equals the residues minus the integral along a semicircle of infinitely large radius $\left(\Gamma_{\infty}^{R}\right.$ or $\left.\Gamma_{\infty}^{L}\right)$, as shown in Fig. 2 , and the latter contribution is zero. It is clear that $\hat{\tau}_{\theta z}(s, \theta)$ and $\hat{\tau}_{r z}(s, \theta)$ are meromorphic functions of $s$ whose poles can occur only at the zeros of $D(s)$.

If $s_{1}$ is a simple zero of $D(s)$, then the stress singularity will be real; thus, if $\lambda$ is the order of the stress singularity, then $\lambda=\operatorname{Re}\left(s_{1}\right)+1$ and for small values of $r$, and the stresses are proportional to $r^{-\lambda}$. If $s_{1}$ is a complex zero, then the stress fields are oscillatory in the limit $r \rightarrow 0$. If no zero of $D(s)$ occurs in $-1<\operatorname{Re}(s)<0$, but $d D(s) / d s=0$ at $s=-1$, then the stresses will have logarithm-type singularity. Hence, determining the location of the zeros of the characteristic function $D(s)$ is our principal task and is used to construct the full-field solutions. It was shown by Ma and Hour (1989) that the zeros of $D(s)$ are always real for any combination of material constants 
and wedge angles, so that the possibility of the oscillatory behavior is precluded. This is a different behavior from the in-plane case.

The theory of residues, is used to obtain the form of the solution and the order of the stress singularity. The general full-field solutions for stresses and displacement in the upper wedge (i.e., $0 \leq \theta \leq \beta$ ) are

$\tau_{\theta z}(r, \theta)=\sum_{i=1}^{\infty} \frac{r^{-s_{i}-1}}{\Psi^{s_{i}}(\theta) \frac{d D\left(s_{i}\right)}{d s}}\left[\hat{t}\left(s_{i}\right) \Psi^{s_{i}}(\beta)\left(K \sin s_{i} \phi_{\alpha} \cos s_{i} \phi\right.\right.$

$\left.\left.+\cos s_{i} \phi_{\alpha} \sin s_{i} \phi\right)+\hat{t}^{*}\left(s_{i}\right) \Psi^{s_{i}}(-\alpha)\left(\sin s_{i} \phi_{\beta} \cos s_{i} \phi-\cos s_{i} \phi_{\beta} \sin s_{i} \phi\right)\right]$

$\tau_{r z}(r, \theta)=\sum_{i=1}^{\infty} \frac{r^{-s_{i}-1}}{\Psi^{s_{i}+2}(\theta) \frac{d D\left(s_{i}\right)}{d s}}\left\{\hat{t}\left(s_{i}\right) \Psi^{s_{i}}(\beta)\left[K \sin s_{i} \phi_{\alpha}\left(\Theta \cos s_{i} \phi\right.\right.\right.$

$\left.\left.-\Omega \sin s_{i} \phi\right)+\cos s_{i} \phi_{\alpha}\left(\Theta \sin s_{i} \phi+\Omega \cos s_{i} \phi\right)\right]$

$+\hat{t}^{*}\left(s_{i}\right) \Psi^{s_{i}}(-\alpha)\left[\sin s_{i} \phi_{\beta}\left(\Theta \cos s_{i} \phi-\Omega \sin s_{i} \phi\right)\right.$

$\left.\left.-\cos s_{i} \phi_{\beta}\left(\Theta \sin s_{i} \phi+\Omega \cos s_{i} \phi\right)\right]\right\}$

$w(r, \theta)=\sum_{i=1}^{\infty} \frac{r^{-s_{i}}}{C s_{i} \Psi^{s_{i}}(\theta) \frac{d D\left(s_{i}\right)}{d s}}\left[\hat{t}\left(s_{i}\right) \Psi^{s_{i}}(\beta)\left(K \sin s_{i} \phi_{\alpha} \sin s_{i} \phi\right.\right.$

$\left.\left.-\cos s_{i} \phi_{\alpha} \cos s_{i} \phi\right)+\hat{t}^{*}\left(s_{i}\right) \Psi^{s_{i}}(-\alpha)\left(\sin s_{i} \phi_{\beta} \sin s_{i} \phi+\cos s_{i} \phi_{\beta} \cos s_{i} \phi\right)\right]$

The general full-field solutions in the lower wedge $(-\alpha \leq \theta \leq 0)$ can also be constructed in a similar manner. The asymptotic behavior of the stress field $\tau_{\theta z}$ as $r \rightarrow 0$ is given by taking only the first term in (37), which yields

$\lim _{r \rightarrow 0} \tau_{\theta z}(r, \theta)=\frac{r^{-s_{1}-1}}{\Psi^{s_{1}}(\theta) \frac{d D\left(s_{1}\right)}{d s}}\left[\hat{t}\left(s_{1}\right) \Psi^{s_{1}}(\beta)\left(K \sin s_{1} \phi_{\alpha} \cos s_{1} \phi\right.\right.$

$\left.\left.+\cos s_{1} \phi_{\alpha} \sin s_{1} \phi\right)+\hat{t}^{*}\left(s_{1}\right) \Psi^{s 1}(-\alpha)\left(\sin s_{1} \phi_{\beta} \cos s_{1} \phi-\cos s_{1} \phi_{\beta} \sin s_{1} \phi\right)\right]$

Eq. (40) shows that the order of the power-type stress singularity is $\lambda=$ $s_{1}+1$, where $s_{1}$ denotes the location of the zero of function $D(s)$ in (36) with the largest value in the open strip $-1<s<0$. It is worth mentioning that the characteristic equation $D(s)$ has the same functional form as the isotropic case if the effective angle $\phi_{\alpha}, \phi_{\beta}$ and the effective material constants $C$ and $C^{*}$ are introduced. It is also interesting to note that the order of stress singularity for an anisotropic bimaterial wedge depends only on one material parameter, $K$, which is the ratio of the effective material constants $C^{*}$ and $C$. We can see from (37) and (39) that solutions for the stress and displacement field have a reduced dependence on the elastic constants. 
Green's Functions for Anisotropic Bimaterial Crack and SINGLE WEDGE

\section{Bimaterial Crack}

There are some special cases in which the zeros of the characteristic equation $D(s)$ can be obtained explicitly. For those special cases, the exact full-field solutions can be expressed explicitly as an infinite series. One such important case is that of an interfacial crack at the interface of two materials. Interfacial cracking is one of the most commonly observed failure modes in fiber-reinforced composite laminates. Thus, the study of interfacial cracks is of great importance in the fracture analysis of composites. We have $\alpha=$ $\pi$ and $\beta=\pi$ for this special case, which implies $\phi_{\alpha}=\phi_{\beta}=\pi$. Suppose that a pair of point loadings with unit magnitude are applied on the crack surfaces with distance $d$ from the crack tip. The traction boundary conditions are given as follows:

$\tau_{\theta z}(r, \pi)=t(r)=\delta(r-d)$

$\tau_{\theta z}^{*}(r,-\pi)=t^{*}(r)=\delta(r-d)$

Where $\delta$ represents the Dirac-delta function. The Mellin transforms of $t(r)$ and $t^{*}(r)$ are $\hat{t}(s)=d^{s}$ and $\hat{t}^{*}(s)=d^{s}$, respectively. The full-field solutions of stresses and displacement in the upper material (i.e., $0 \leq \theta \leq \pi$ ) for a bimaterial crack geometry are given by the following expressions:

$\tau_{\theta z}(r, \theta)=\frac{\Psi(\theta)}{d \pi} \sum_{n=0}^{\infty}(-1)^{n} \cos \frac{2 n+1}{2} \phi\left[\frac{r \Psi(\theta)}{d}\right]^{(2 n+1) / 2-1}$

$\cdot H[d-r \Psi(\theta)]+\frac{\Psi(\theta)}{d \pi} \sum_{n=0}^{\infty}(-1)^{n}$

$\cdot \cos \frac{2 n+1}{2} \phi\left[\frac{d}{r \Psi(\theta)}\right]^{(2 n+1) / 2+1} H[r \Psi(\theta)-d]$

$\tau_{\theta z}(r, \theta)=\frac{\Psi(\theta)}{d \pi} \sqrt{\frac{d}{r \Psi(\theta)}}\left\{\frac{\left[1+\frac{r \Psi(\theta)}{d}\right] \cos \frac{\phi}{2}}{\left[\frac{r \Psi(\theta)}{d}\right]^{2}+2\left[\frac{r \Psi(\theta)}{d}\right] \cos \phi+1}\right\}$

$\tau_{r z}(r, \theta)=\frac{1}{\Psi(\theta) d \pi}\left\{\Theta \sum_{n=0}^{\infty}(-1)^{n} \cos \frac{2 n+1}{2} \phi\left[\frac{r \Psi(\theta)}{d}\right]^{(2 n+1) / 2-1}\right.$

$\left.+\Omega \sum_{n=0}^{\infty}(-1)^{n} \sin \frac{2 n+1}{2} \phi\left[\frac{r \Psi(\theta)}{d}\right]^{(2 n+1) / 2-1}\right\} H[d-r \Psi(\theta)]$

$+\frac{1}{\Psi(\theta) d \pi}\left\{\Theta \sum_{n=0}^{\infty}(-1)^{n} \cos \frac{2 n+1}{2} \phi\left[\frac{d}{r \Psi(\theta)}\right]^{(2 n+1) / 2+1}\right.$

$\left.-\Omega \sum_{n=0}^{\infty}(-1)^{n} \sin \frac{2 n+1}{2} \phi\left[\frac{d}{r \Psi(\theta)}\right]^{(2 n+1) / 2+1}\right\} H[r \Psi(\theta)-d] \ldots$ 


$$
\begin{aligned}
& w(r, \theta)=\frac{1}{C \pi} \sum_{n=0}^{\infty}(-1)^{n}\left(\frac{2}{2 n+1}\right) \sin \left(\frac{2 n+1}{2} \phi\right) \\
& \cdot\left[\frac{r \Psi(\theta)}{d}\right]^{(2 n+1) / 2} H[d-r \Psi(\theta)]+\frac{1}{C \pi} \sum_{n=0}^{\infty}(-1)^{n} \\
& \cdot\left(\frac{2}{2 n+1}\right) \sin \left(\frac{2 n+1}{2} \phi\right)\left[\frac{d}{r \Psi(\theta)}\right]^{(2 n+1) / 2} H[r \Psi(\theta)-d] \\
& w(r, \theta)=\frac{1}{2 C \pi} \ln \left[\frac{\left.1+2 \sqrt{\frac{r \Psi(\theta)}{d}} \sin \frac{\phi}{2}+\frac{r \Psi(\theta)}{d}\right] \ldots}{\left.1-2 \sqrt{\frac{r \Psi(\theta)}{d}} \sin \frac{\phi}{2}+\frac{r \Psi(\theta)}{d}\right] \ldots}\right.
\end{aligned}
$$

where $H$ is the Heaviside unit step function. The solutions of stresses and displacement in the lower material (i.e., $-\pi \leq \theta \leq 0$ ) are obtained by replacing $\Psi(\theta), \phi, \Theta, \Omega$, and $C$ by $\Psi^{*}(\theta), \phi^{*}, \Theta^{*}, \Omega^{*}$, and $C^{*}$, respectively in $(42 a)-(44 b)$. Note that the exact full-field solutions of stresses and displacement in the upper material (42) - (44) for the bimaterial crack problem depend only on the material properties $c_{44}, c_{45}$, and $c_{55}$ in the upper part. Furthermore, the solutions (42)-(44) is exactly the same as the problem for a homogeneous anisotropic material containing a crack. Along the interface $\theta=0$ the stresses and displacement are

$$
\begin{aligned}
& \tau_{\theta z}(r, 0)=\tau_{\theta z}^{*}(r, 0)=\frac{1}{d \pi} \frac{\frac{d}{r}}{\left(\frac{d}{r}\right)^{1 / 2}+\left(\frac{r}{d}\right)^{1 / 2}} \ldots \ldots \ldots \ldots \ldots \ldots \ldots \\
& \tau_{r z}(r, 0)=\frac{1}{d \pi} \frac{c_{45}}{c_{44}} \frac{\frac{d}{r}}{\left(\frac{d}{r}\right)^{1 / 2}+\left(\frac{r}{d}\right)^{1 / 2}} \\
& \tau_{r z}^{*}(r, 0)=\frac{1}{d \pi} \frac{\frac{d}{r}}{c_{45}^{*}} \frac{\left.\frac{d}{r}\right)^{1 / 2}+\left(\frac{r}{d}\right)^{1 / 2}}{(r)} \\
& w(r, 0)=w^{*}(r, 0)=0
\end{aligned}
$$

As shown in (45)-(48), the shear stress $\tau_{\theta z}$ is continuous on the bonded interface; while $\tau_{r z}$ is discontinuous. Although the stress field of the loaded composite is influenced by the elastic constants of the bonded materials, the stress $\tau_{\theta z}$ at the interface ahead of the interface crack [(45)] can be described independently of the elastic composite parameters and the solution is the same as that for isotropic bimaterial crack. The asymptotic behavior of the stresses and displacement for very small values of $r$ can be described in the following form: 
$\lim _{r \rightarrow 0} \tau_{\theta z}(r, \theta)=\frac{1}{\pi} \sqrt{\frac{\Psi(\theta)}{d r}} \cos \frac{\phi}{2}$

$\lim _{r \rightarrow 0} \tau_{r z}(r, \theta)=\frac{1}{\pi} \sqrt{\frac{1}{\Psi^{3}(\theta) d r}}\left[\Theta \cos \frac{\phi}{2}+\Omega \sin \frac{\phi}{2}\right]$

$\lim _{r \rightarrow 0} w(r, \theta)=\frac{2}{C \pi} \sqrt{\frac{r \Psi(\theta)}{d}} \sin \frac{\phi}{2}$

The material constants chosen for the numerical investigation are $c_{45} / c_{44}$ $=0.8 ;$ and $c_{55} / c_{44}=4$. The values of $\phi$ and $\Psi$ as function of $\theta$ are presented in Fig. 3. Some numerical results of the full-field solution for stresses in the upper material are presented based on the analytical solution obtained for interfacial crack. In Fig. 4, we can see that the shear stress $\tau_{\theta z}$ increases as $r / d$ decreases for $\theta \leq \pi / 2$, but for $r / d \rightarrow 1$ the stress increases very rapidly near the crack face, which shows the influence from the point load at $r=$ $d$. Fig. 5 shows the solution of shear stress $\tau_{\theta z}$ for fixed $\theta$, the stress behaves with square-root singularity as $r / d \rightarrow 0$, and decreases very rapidly and tends to zero as $r / d$ become large. Fig. 6 shows the angular variation of $\tau_{r \theta}$ for fixed $r / d$; the stress is not zero at the crack face and it indicates the singular behavior at the crack face as $r / d$ approaches 1 . Fig. 7 also shows the squareroot singular behavior as $r / d \rightarrow 0$, and the stress tends to zero as $r / d$ become large. For the isotropic bimaterial crack, we have $c_{45}=0$; and $c_{44}=c_{55}=$ $\mu$, which gives $C=\mu ; \Psi(\theta)=1 ; \phi=\theta ; \Theta=0$; and $\Omega=1$. Then the solutions represented in (42)-(49) reduce to the solutions obtained by $\mathrm{Ma}$ (1989) for isotropic material.

\section{Single Wedge}

We now consider that a single wedge with wedge angle $\alpha+\beta$ subjected to a pair of concentrate point loading. We have $c_{44}^{*}=c_{44} ; c_{45}^{*}=c_{45}$; and $c_{55}^{*}=c_{55}$; then the full-field solutions of this problem can be obtained from (37) $-(39)$ as follows:

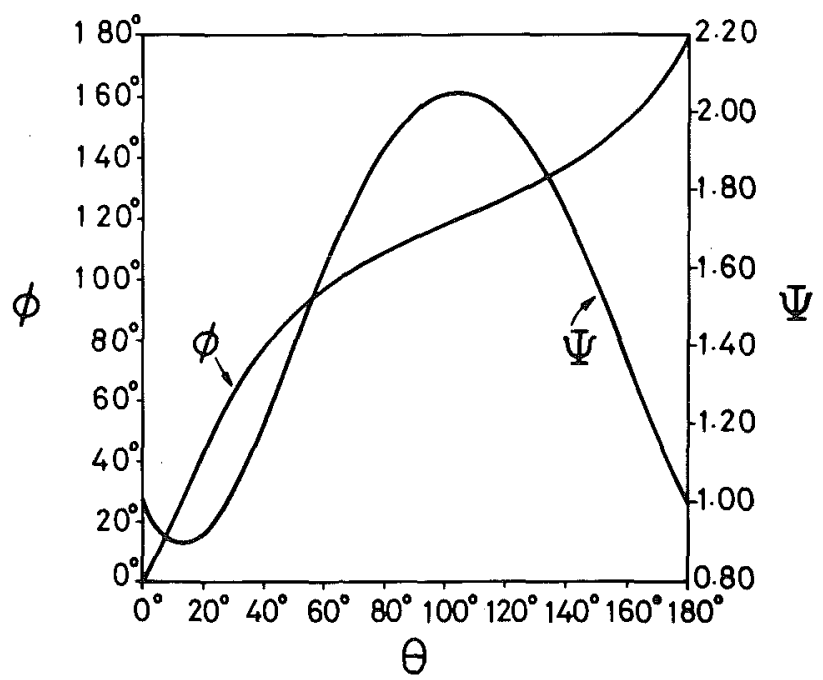

FIG. 3. Numerical Results of $\phi$ and $\Psi$ as Function of $\theta$ for $c_{45} / c_{44}=0.8 ; c_{55} / c_{44}=4$ 


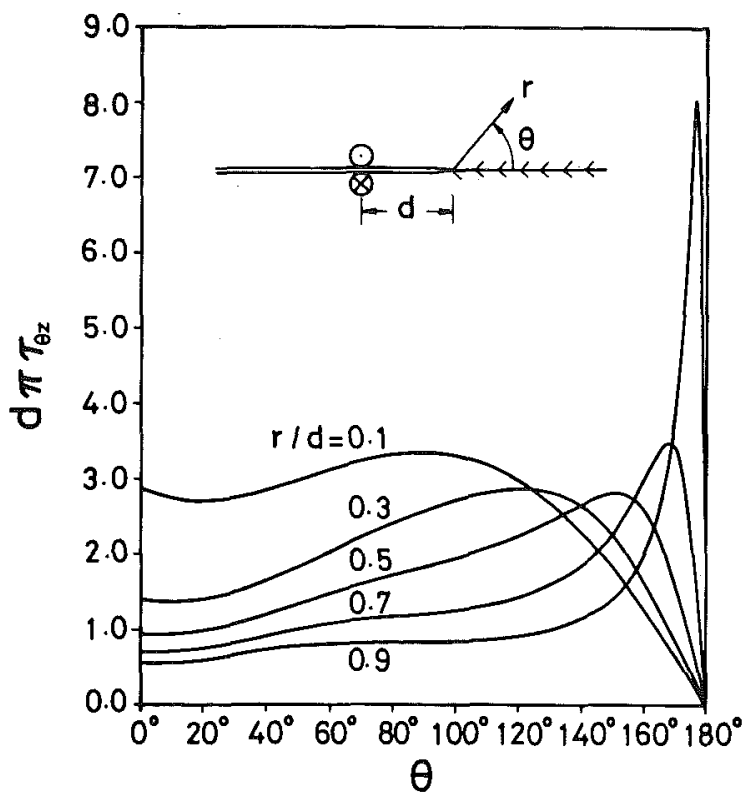

FIG. 4. Full-Field Solution of Shear Stress $\tau_{\theta z}$ for Fixed $r$ of Bimaterial Anisotropic Crack

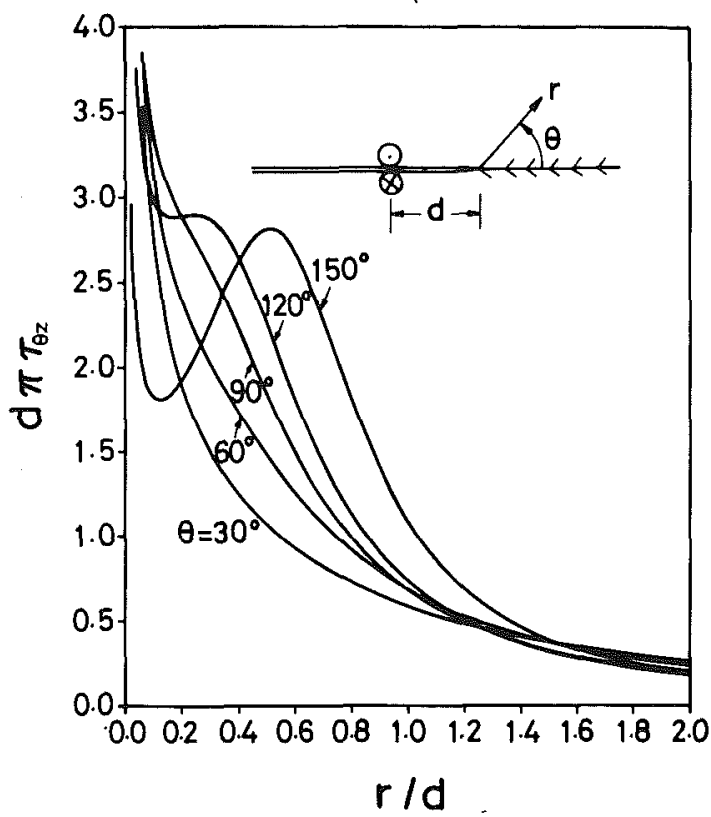

FIG. 5. Full-Field Solution of Shear Stress $\tau_{\theta z}$ for Fixed $\theta$ of Bimaterial Anisotropic Crack 


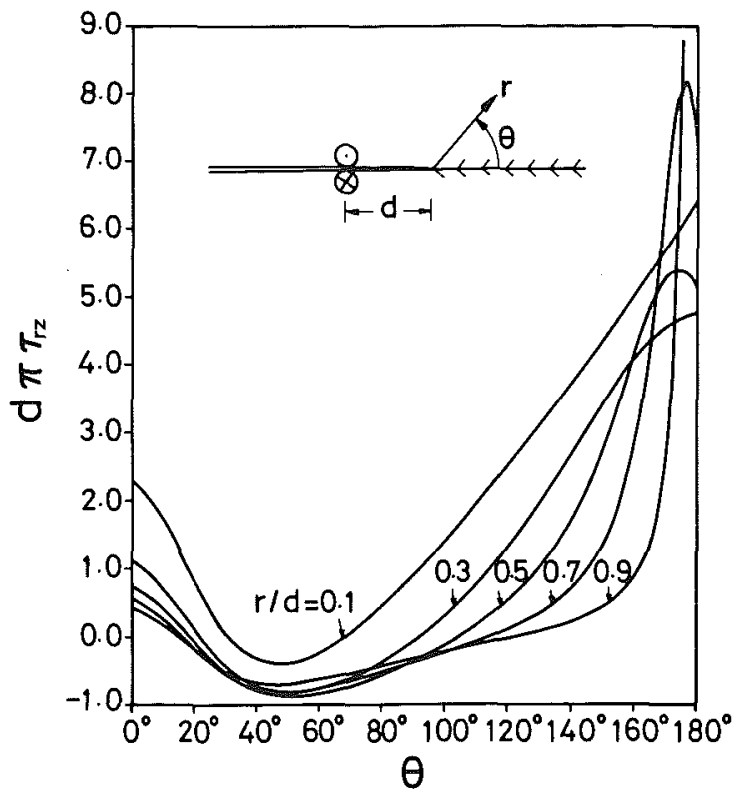

FIG. 6. Full-Field Solution of Shear Stress $\tau_{r z}$ for Fixed $r$ of Bimaterial Anisotropic Crack

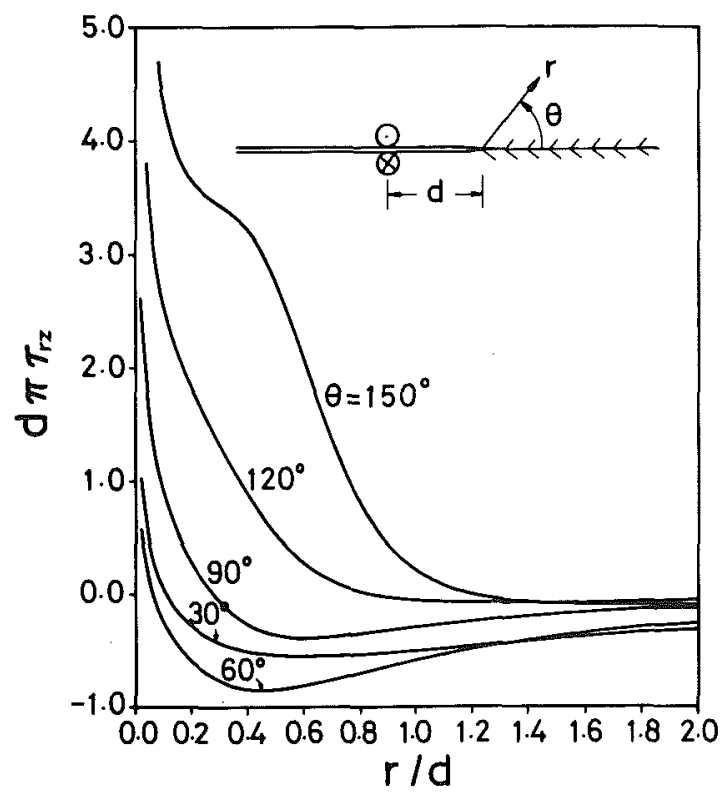

FIG. 7. Full-Field Solution of Shear Stress $\tau_{r z}$ for Fixed $\theta$ of Bimaterial Anisotropic Crack 


$$
\begin{aligned}
& \tau_{\theta z}(r, \theta)=-\frac{\Psi(\theta)}{d \Phi}\left\{\frac{1}{\Psi(\beta)} \sum_{n=1}^{\infty}(-1)^{n} \sin \frac{n \pi}{\Phi}\left(\phi_{\alpha}+\phi\right)\right. \\
& \cdot\left[\frac{r \Psi(\theta)}{d \Psi(\beta)}\right]^{(n \pi / \Phi)-1} H[d \Psi(\beta)-r \Psi(\theta)]+\frac{1}{\Psi(\beta)} \sum_{n=1}^{\infty}(-1)^{n} \\
& \cdot \sin \frac{n \pi}{\Phi}\left(\phi_{\alpha}+\phi\right)\left[\frac{d \Psi(\beta)}{r \Psi(\theta)}\right]^{(n \pi / \Phi)+1} H[r \Psi(\theta)-d \Psi(\beta)] \\
& +\frac{1}{\Psi(-\alpha)} \sum_{n=1}^{\infty}(-1)^{n} \sin \frac{n \pi}{\Phi}\left(\phi_{\beta}-\phi\right)\left[\frac{r \Psi(\theta)}{d \Psi(-\alpha)}\right]^{(n \pi / \Phi)-1} \\
& H[d \Psi(-\alpha)-r \Psi(\theta)]+\frac{1}{\Psi(-\alpha)} \sum_{n=1}^{\infty}(-1)^{n} \\
& \left.\cdot \sin \frac{n \pi}{\Phi}\left(\phi_{\beta}-\phi\right)\left[\frac{d \Psi(-\alpha)}{r \Psi(\theta)}\right]^{(n \pi / \Phi)+1} H[r \Psi(\theta)-d \Psi(-\alpha)]\right\} \\
& \tau_{r z}(r, \theta)=-\frac{1}{\Psi(\theta) d \Phi}\left\{\frac { 1 } { \Psi ( \beta ) } \sum _ { n = 1 } ^ { \infty } ( - 1 ) ^ { n } \left[\Theta \sin \frac{n \pi}{\Phi}\left(\phi_{\alpha}+\phi\right)\right.\right. \\
& \left.-\Omega \cos \frac{n \pi}{\Phi}\left(\phi_{\alpha}+\phi\right)\right]\left[\frac{r \Psi(\theta)}{d \Psi(\beta)}\right]^{(n \pi / \Phi)-1} \\
& \cdot H[d \Psi(\beta)-r \Psi(\theta)]+\frac{1}{\Psi(\beta)} \sum_{n=0}^{\infty}(-1)^{n}\left[\Theta \sin \frac{n \pi}{\Phi}\left(\phi_{\alpha}+\phi\right)\right. \\
& \left.+\Omega \cos \frac{n \pi}{\Phi}\left(\phi_{\alpha}+\phi\right)\right]\left[\frac{d \Psi(\beta)}{r \Psi(\theta)}\right]^{(n \pi / \dot{\alpha})+1} \\
& \cdot H[r \Psi(\theta)-d \Psi(\beta)]+\frac{1}{\Psi(-\alpha)} \sum_{n=1}^{\infty}(-1)^{n}\left[\Theta \sin \frac{n \pi}{\Phi}\left(\phi_{\beta}-\phi\right)\right. \\
& \left.+\Omega \cos \frac{n \pi}{\Phi}\left(\phi_{\beta}-\phi\right)\right]\left[\frac{r \Psi(\theta)}{d \Psi(-\alpha)}\right]^{(n \pi / \Phi)-1} H[d \Psi(-\alpha)-r \Psi(\theta)] \\
& +\frac{1}{\Psi(-\alpha)} \sum_{n=0}^{\infty}(-1)^{n}\left[\Theta \sin \frac{n \pi}{\Phi}\left(\phi_{\beta}-\phi\right)-\Omega \cos \frac{n \pi}{\Phi}\left(\phi_{\beta}-\phi\right)\right] \\
& \left.\cdot\left[\frac{d \Psi(-\alpha)}{r \Psi(\theta)}\right]^{(n \pi / \Phi)+1} H[r \Psi(\theta)-d \Psi(-\alpha)]\right\}
\end{aligned}
$$




$$
\begin{aligned}
& w(r, \theta)=\frac{1}{C \Phi}\left\{\sum_{n=1}^{\infty}(-1)^{n}\left(\frac{\Phi}{n \pi}\right) \cos \frac{n \pi}{\Phi}\left(\phi_{\alpha}+\phi\right)\right. \\
& \cdot\left[\frac{r \Psi(\theta)}{d \Psi(\beta)}\right]^{n \pi / \Phi} H[d \Psi(\beta)-r \Psi(\theta)] \\
& +\left(\sum_{n=1}^{\infty}(-1)^{n}\left(\frac{\Phi}{n \pi}\right) \cos \frac{n \pi}{\Phi}\left(\phi_{\alpha}+\phi\right)\left[\frac{d \Psi(\beta)}{r \Psi(\theta)}\right]^{n \pi / \Phi}\right. \\
& \left.+\ln \left[\frac{d \Psi(\beta)}{r \Psi(\theta)}\right]\right) H[r \Psi(\theta)-d \Psi(\beta)] \\
& -\sum_{n=1}^{\infty}(-1)^{n}\left(\frac{\Phi}{n \pi}\right) \cos \frac{n \pi}{\Phi}\left(\phi_{\beta}-\phi\right) \\
& .\left[\frac{r \Psi(\theta)}{d \Psi(-\alpha)}\right]^{n \pi / \Phi} H[d \Psi(-\alpha)-r \Psi(\theta)] \\
& -\left(\sum_{n=1}^{\infty}(-1)^{n}\left(\frac{\Phi}{n \pi}\right) \cos \frac{n \pi}{\Phi}\left(\phi_{\beta}-\phi\right)\left[\frac{d \Psi(-\alpha)}{r \Psi(\theta)}\right]^{n \pi / \Phi}\right. \\
& \left.\left.+\ln \left[\frac{d \Psi(-\alpha)}{r \Psi(\theta)}\right]\right) H[r \Psi(\theta)-d \Psi(-\alpha)]\right\} \ldots
\end{aligned}
$$

where $\Phi=\phi_{\alpha}+\phi_{\beta}$. The asymptotic stress and displacement fields near the single anisotropic wedge apex are

$$
\begin{aligned}
& \lim _{r \rightarrow 0} \tau_{\theta z}(r, \theta)=\frac{r^{\pi / \Phi-1}}{\Phi}\left\{\sin \frac{\pi}{\Phi}\left(\phi_{\alpha}+\phi\right)\left[\frac{\Psi(\theta)}{d \Psi(\beta)}\right]^{\pi / \Phi}\right. \\
& \left.+\sin \frac{\pi}{\Phi}\left(\phi_{\beta}-\phi\right)\left[\frac{\Psi(\theta)}{d \Psi(-\alpha)}\right]^{\pi / \Phi}\right\} \ldots \ldots \ldots \ldots \ldots \ldots \ldots \\
& \lim _{r \rightarrow 0} \tau_{r z}(r, \theta)=\frac{r^{\pi / \Phi-1}}{\Phi \Psi^{2}(\theta)}\left\{\left[\Theta \sin \frac{\pi}{\Phi}\left(\phi_{\alpha}+\phi\right)\right.\right. \\
& \left.-\Omega \cos \frac{\pi}{\Phi}\left(\phi_{\alpha}+\phi\right)\right]\left[\frac{\Psi(\theta)}{d \Psi(\beta)}\right]^{\pi / \Phi} \\
& \left.+\left[\Theta \sin \frac{\pi}{\Phi}\left(\phi_{\beta}-\phi\right)+\Omega \cos \frac{\pi}{\Phi}\left(\phi_{\beta}-\phi\right)\right]\left[\frac{\Psi(\theta)}{d \Psi(-\alpha)}\right]^{\pi / \Phi}\right\} \\
& \lim _{r \rightarrow 0} w(r, \theta)=\frac{r^{\pi / \Phi}}{C \pi}\left\{-\cos \frac{\pi}{\Phi}\left(\phi_{\alpha}+\phi\right)\left[\frac{\Psi(\theta)}{d \Psi(\beta)}\right]^{\pi / \Phi}\right. \\
& \left.+\cos \frac{\pi}{\Phi}\left(\phi_{\beta}-\phi\right)\left[\frac{\Psi(\theta)}{d \Psi(-\alpha)}\right]^{\pi / \Phi}\right\} \ldots \ldots
\end{aligned}
$$




\section{Concluding Remarks}

A study of the antiplane shear problem was presented. The Mellin transform method was developed to obtain the general solution for the governing partial differential equation. Full-field solutions for the stresses and deformation fields in the anisotropic bimaterial wedge problem are constructed from the homogeneous solution in the form of infinite series. Based on the complete solution obtained, the asymptotic solution for the singular stress field near the wedge apex is expressed. The complete solutions for a point load along the anisotropic bimaterial crack face and single anisotropic wedge are presented and therefore can be used to generate the solution for other problems with traction boundary conditions. It is worth mentioning that the order of stress singularity for a bimaterial wedge depends only on one material parameter, and the stress and displacement fields have a reduced dependence on the elastic constants. Furthermore, the stress and displacement solutions in the upper material for interfacial crack problem depend only on the material constants in the upper part. Along the interface of the interfacial crack, the shear stress $\tau_{\mathrm{gz}}$ is independent of the elastic constants, and it is the same as that for an isotropic interfacial crack.

The bimaterial problems for cracks and wedges have been extensively studied in the technical literature for isotropic and anisotropic bonded materials of in-plane deformation. The linear elasticity solutions predict that the order of stress singularity is a complex number, which implies the physically disagreeable result of oscillations in stresses and displacements. However, Ma and Hour (1989) found that the order of the stress singularity is always real and precludes this physically unacceptable feature in the inplane case.

In real structures, the geometry is generally too complex to allow analytical solution. Therefore, one has to resort to numerical techniques, such as the finite element method. Because the stress state is singular near the bimaterial wedge apex, numerical results obtained by ignoring the singularity can be erroneous. Therefore, special elements must be designed and incorporated into the finite element grid near a singular stress field. The asymptotic expressions and some exact solutions given in this paper can be used to formulate those special elements and to check the accuracy of the numerical results.

\section{ACKNOWLEDGMENTS}

The writer is grateful to $\mathrm{K}$. C. Wu for reading the manuscript. The financial supported by the National Science Council (Republic of China) through grant NSC 79-0401-E-002-37 to the National Taiwan University is gratefully acknowledged.

\section{Appendix. References}

Atkinson, C. (1977). "On stress singularities and interfaces in linear elastic fracture mechanics." Int. J. Fract., 13(6), 807-820.

Bogy, D. B. (1971). "Two edge-bonded elastic wedges of different materials and wedge angles under surface tractions." J. Appl. Mech., 38(2), 377-386.

Bogy, D. B. (1972). "The plane solution for anisotropic elastic wedges under normal and shear loading." J. Appl. Mech., 39(4), 1103-1109.

Comninou, M. (1977). "The interface crack." J. Appl. Mech., 44(4), 631-636. 
Dundurs, J. (1967). "Effect of elastic constants on stress in a composite under plane deformation." J. Composite Mat., 1, 310-322.

England, A. H. (1965). "A crack between dissimilar media." J. Appl. Mech., 32(2), $400-402$.

Erdogan, F. (1963). "Stress distribution in a nonhomogeneous elastic plane with cracks." J. Appl. Mech., 30(2), 232-236.

Gotoh, H. (1967). "Some problems of bonded anisotropic plates with cracks along the bond." Int. J. Fract., 3(4), 253-265.

$\mathrm{Ma}, \mathrm{C}$. C. (1989). "Analysis of the green functions for the antiplane dissimilar isotropic wedges with equal wedge angles." Bulletin of the Coll. of Engrg., National Taiwan University, Taipei, Taiwan, 46(Jun.), 69-81.

Ma, C. C., and Hour, B. L. (1989). "Analysis of dissimilar anisotropic wedges subjected to antiplane shear deformation." Int. J. Solids Struct., 25(11), 12951309.

Ma, C. C., and Wu, H. W. (1990). "Analysis of inplane composite wedges under traction-displacement or displacement-displacement boundary conditions." Acta Mechanica, 85(3), 149-167.

Qu, J., and Bassani, J. L. (1989). "Cracks on bimaterial and bicrystal interfaces." J. Mech. Phys. Solids, 37(4), 417-433.

Rice, J. R., and Sih, G. C. (1965). "Plane problems of cracks in dissimilar media." J. Appl. Mech., 32(2), 418-423.

Stroh, A. N. (1958). "Dislocations and cracks in anisotropic elasticity." Phil. Mag., $7,625-646$.

Ting, T.C.T. (1986). "Explicit solution and invariance of the singularities at an interface crack in anisotropic composites." Int. J. Solids Struct., 22(9), 965-983.

Tranter, C. J. (1948). "The use of the Mellin transform in finding the stress distribution in an infinite wedge." $Q$. J. Mech. Appl. Math., 1(2), 125-130.

Williams, M. L. (1952). "Stress singularities resulting from various boundary conditions in angular corners of plates in extension." J. Appl. Mech., 19(4), 526-528.

Williams, M. L. (1959). "The stresses around a fault of crack in dissimilar media." Bulletin Seis. Soc. Am., 49(2), 199-204.

Willis, J. R. (1971). "Fracture mechanics of interfacial cracks." J. Mech. Phys. Solids, 19(6), 353-368.

$\mathrm{Wu}, \mathrm{K}$. C. (1990). "Stress intensity factor and energy release rate for interfacial cracks between dissimilar anisotropic material." J. Appl. Mech., 57(4), 882-886.

Wu, K. C. (1991). "Anti-plane shear interface in anisotropic bimaterials." J. Appl. Mech. , 58(2), 399-403. 